\title{
The Feasibility of Ultrasound to Assess Structural Anatomy of the Larynx
}

\author{
Sharma Nitu ${ }^{1,2}$, Gnawali Suman ${ }^{1}$, Poudel Shivaji ${ }^{3}$, Lino Santiago $S$ \\ Pabillo $^{2}$, Chila Deguito ${ }^{2}$ and Yadav Ajay Kumar ${ }^{1 *}$ \\ ${ }^{1}$ Department of Radio-Diagnosis, Imaging and Nuclear Medicine, BP Koirala \\ Memorial Cancer Hospital, Bharatpur, Chitwan, Nepal \\ ${ }^{2}$ Department of Medical Imaging, Quirino Memorial Medical Center (QMMC), \\ Manila, Philippines \\ ${ }^{3}$ Department of Radiation Oncology, BP Koirala Memorial Cancer Hospital, \\ Bharatpur, Chitwan, Nepal \\ *Corresponding Author: Yadav Ajay Kumar, Department of Radio-Diagnosis, \\ Imaging and Nuclear Medicine, BP Koirala Memorial Cancer Hospital, Bharatpur, \\ Chitwan, Nepal.
}

Received: August 18, 2021

Published: September 17, 2021

(C) All rights are reserved by Sharma Nitu., et

al.

\begin{abstract}
Ultrasonography (USG) has been proven to be a fast, easily available, and cost-effective diagnostic tool in evaluating various organs of the body, however, its use in the evaluation of the larynx has not been popular. In this cross-sectional quantitative study 71 healthy volunteer, 20 - 40 years of age got ultrasound with linear transducer (7 - $13 \mathrm{MHz}$ ) in B-mode. Obtained data were analyzed using SPSS ver.20 software and shown in frequency, percentages and bar diagram. The mean length of Laryngeal Inlet, Glottis and Tracheal Air Column is 1.049, 0.630, and 1.131 respectively in female. In male the mean length of Laryngeal Inlet, Glottis and Tracheal Air Column is 1.22, 0.70 and $1.34 \mathrm{~cm}$ respectively. High frequency linear transducer has proven to be most appropriate in evaluating the larynx. This study has revealed the potential utility of ultrasound to assess the structural anatomy of larynx specially focusing at the hyoid bone level, thyroid cartilage level and cricoids cartilage level and creating normal values of laryngeal inlet, glottis opening and tracheal air column through sonography which can be used in various clinical applications. The measurement gained during this study was proven to be easily reproducible.
\end{abstract}

Keywords: USG; Glottis; Laryngeal Inlet; Tracheal Air Column

\section{Introduction}

Endoscopy, Computed Tomography (CT) and Magnetic Resonance Imaging (MRI) have been backbone within the assessment of the larynx and its encompassing structures. Ultrasonography has been demonstrated to be a quick, effectively accessible, and cost-effective symptomatic apparatus in assessing different organs of the body, however, its utilize within the assessment of the larynx has not been well known. This exclusion is related to the issue of visualization of laryngeal structures and hence performing a total laryngeal Ultrasonography examination due to their little estimate and discuss within the gap. But this impediment and other ones were as of late overcome and fathomed by the progress in USG machines and respiratory strategies utilized amid examination. Straightforwardness of this examination, no radiation introduction of the understanding and staff, and the need 
of torment and other negative impacts on the quiet are impressive focal points which pushes this strategy to adopt.

Otolaryngologists utilize diverse demonstrative approaches in assessment of patients with laryngeal injury, and no positive clear agreement have been drawn nearer as respect the arrangement of work up for those patients. However total nitty gritty history with through physical examination more often than not uncovered the etiology of the conclusion in most of the patients.

Wide assortments of procedures to evaluate laryngeal work are accessible. These strategies can for the most part be partitioned into clinical and investigational categories. A down to earth procedure ideally ought to be secure, non-invasive, well-tolerated, and dependable. The outcomes about ought to be reproducible, exact, and recordable for future reference [1].

CT and MRI are profitable symptomatic instruments for genuine and untrue vocal folds pathology however with concern of radiation presentation in CT. With MRI, imaging times are moderately long, and respiratory, gulping, and other movement artifacts can degrade picture quality [2].

Utilizing unbending laryngoscope may lead to over- and underdiagnosis of vocal overlap of motion due to the truth of splinting of the larynx, destitute visualization due to supraglottic collapse, and obsession of cricoarytenoid and dumbfounding developments of vocal overlay coming about from the respiratory glottic discuss stream (Bernoulli impact) [3]. Ultrasonography is routinely done for understanding with head and neck tumors to identify cervical lymph nodal metastases, in spite of the fact that later ponders appears promising outcomes about as respect utilize of US innovation for determination and arranging laryngeal neoplasm however so distant it isn't routinely utilized. Ultrasound is predominant to CT and MRI in assessment of vocal cord motility [4].

It was considered that laryngoscopy was the brilliant standard test of vocal fold mobility, recurrent laryngeal nerve damage, anatomical and useful variety after, and/or some time recently cervical or thoracic surgery. However, it forces additional costs (budgetary burden), time-consuming, and is unsavory involvement for most patients [5].

Ultrasonography could be a cost-effective, secure, fast, effortless, broadly accessible, well-tolerated, not requiring sedation, non-invasive, and non-irradiating strategy with precision comparable to that of laryngoscopy; it can be utilized as a valuable aide to endoscopy-the current gold standard-especially in children [3].

Portable ultrasound gadgets are presently more accessible, reasonable, and of tall demonstrative esteem due to the sensational innovation enhancement. These advances presently offer otolaryngologists a versatile apparatus that increments symptomatic exactness, encourages commonly performed strategies, and includes to understanding comfort and security. Given the information of head and neck life structures that otolaryngologist has, including ultrasound to an otolaryngology practice is getting to be so much simpler and making much more sense [6].

\section{Aim of the Study}

The aim of our study was to appraise the laryngeal ultrasound as a diagnostic tool to assess different laryngeal structure as regard laryngeal dynamics (range and abnormality), and anatomic structures.

\section{Method and Population}

This is a descriptive study done in the Philippine setting most specifically in Quirino Memorial Medical Center (QMMC) of 71 healthy volunteer, 20 - 40 years of age

\section{Materials used}

HITACHI HI VISION AVIUS mid-range ultrasound machine; using a linear transducer (7 - $13 \mathrm{MHz}$ ) in B-mode [1].

SPSS version 20 and Microsoft Excel for statistical analysis.

\section{Sampling size and technique}

4 months of sample collection with, non-random purposive sampling

$\mathrm{n}=\mathrm{Z} \alpha^{2} \mathrm{pq} / \mathrm{d}^{2}$

Where, $\mathrm{n}=$ Number of samples

$\mathrm{p}=$ Proportion or prevalence $=0.4$

$\mathrm{q}=1-\mathrm{p}=0.6$

$\mathrm{d}=$ Margin of error (the precision) $=10 \%$ and $\mathrm{Z} \alpha=1.96$

$(1.96)^{2} \times 0.4 \times 0.6 /(0.11)^{2}=76.19 \infty 71$. 


\section{Data collection method}

Volunteer were informed about the procedure and after their informed written consent. The ultrasound transducer was oriented transversely across the anterior surface of the neck (the transverse view) and was placed at 3 separate locations.

Ultrasonography scanning technique was adopted from Singh M., et al. (2010) to evaluate feasibility of ultrasound to assess structural anatomy of the larynx [7]. The sonographic findings were quantified to determine the dimensions of the following (in centimeters): 1) antero-posterior diameter of the laryngeal inlet at hyoid bone level (Figure 1); 2) transverse diameter of the laryngeal air-column during glottic opening at the thyroid cartilage level during phonation ("ee-ee") to distinguish the true from the false vocal cords (Figure 2 and 3) and 3) transverse diameter of tracheal air column at the cricoids arch level (Figure 4). Each patient was assigned a personal case number after getting consent from them. Three trials were done for each parameter and the measurements were recorded by the primary author and all the measurements, including the average/final measurements were approved by two certified ultrasonologist [7].

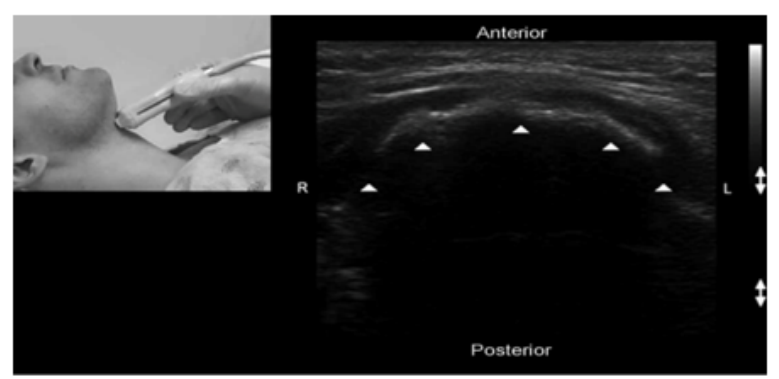

Figure 1: Sonogram of the hyoid bone on a midline transverse view using a linear transducer, the hyoid bone is shown as an inverted U hyperechoic curvilinear line (arrowheads) with posterior acoustic shadowing. The inset shows the transducer position on the skin [7].

Inclusion criteria: No previous or present subjective complaints pertaining to or relating to the larynx, no known history of any laryngeal disorder.

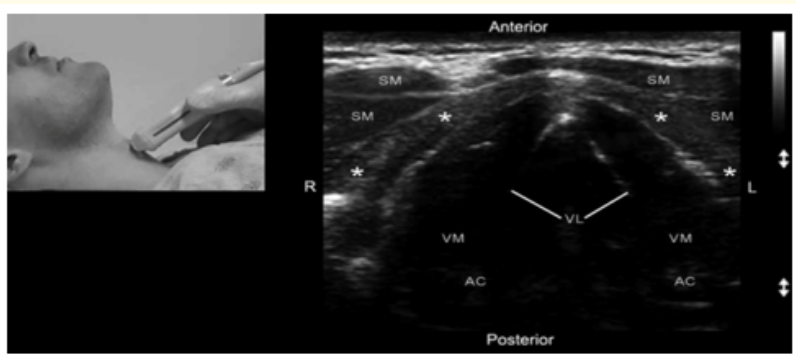

Figure 2: Transverse scan over the thyroid cartilage using a linear transducer, the sonogram shows the true vocal cords and the vocal ligaments near the anterior commissure. The inset shows the transducer position on the skin. AC indicates arytenoids cartilage; asterisks, thyroid cartilage; SM, strap muscles; VL, vocal ligaments, and VM, vocalis muscle [7].

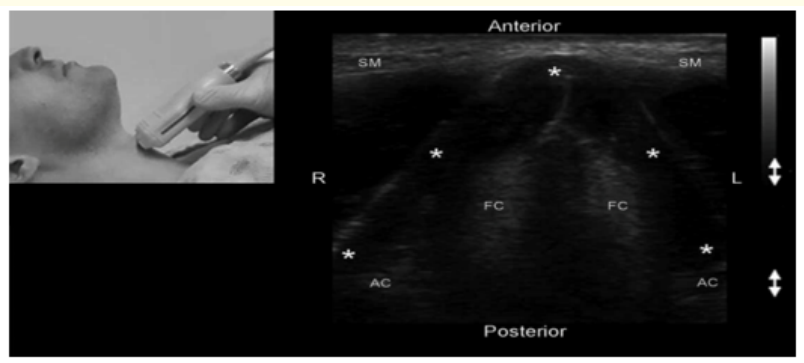

Figure 3: Transverse scan at the level of the thyroid cartilage using a linear transducer. The sonogram shows the hyperechoic false cords (FC). Note that the echogenicity of the true and false cords is different because of the increased fat content in the false cords and the presence of the vocalis muscle in the true cords. The inset shows the transducer position on the skin. AC indicates arytenoid cartilage; and asterisks, thyroid cartilage [7].
Exclusion criteria: Exclusion criteria included history of surgical intervention/manipulation of the neck (i.e., video laryngoscopy), history of intubation of at least 3 weeks and findings of structural abnormalities of the landmarks that will be used during scanning. 


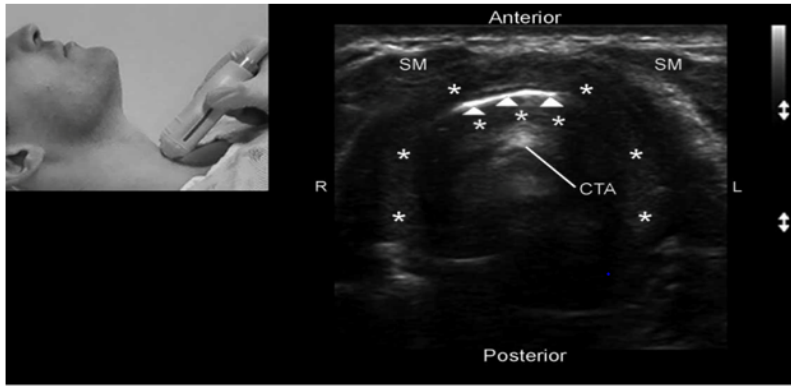

Figure 4: Transverse scan at the level of the cricoids cartilage using a linear transducer. The sonogram shows the cricoids cartilage with a posterior A-M interface (arrowheads) and comet tail artifacts (CTA). The inset shows the transducer position on the skin. Center asterisks indicate reverberation artifacts; left and right asterisks, cricoids cartilage; and SM, strap muscle [7].

Ethical consideration: Approval was taken from Institutional Review Board of the data collecting hospital; Informed consent was taken from the participant.

Data analysis: Statistical analysis was carried out with the help of SPSS version 20 and Microsoft Excel. Factor and percentages were calculated. Graphical representations were done through line diagrams.

\section{Result}

\section{Profile of respondents}

Results reveal that most of the study patients are female (57.6\%) while $42.4 \%$ are male. Results further show that majority of them are from 20 to 30 years old (83.3\%), while $16.7 \%$ are from 31 to 40 years old. Moreover, results show that among male study patients, $82.1 \%$ of them from 20 to 30 years old, while among female, $82.21 \%$ are also from that same age group which is summarized in table 1, table 2 and graphical representation is shown in figure 5 and 6.

Results show that the average laryngeal inlet of female patients aged 20 to 30 years old is $1.027 \pm 0.155$ and a median of 0.988 . Moreover, the mean glottis is $0.620 \mathrm{~cm} \pm 0.098$ while the resulting mean tracheal air column turns out to be $1.119 \mathrm{~cm}$.

\begin{tabular}{|l|c|c|}
\hline Profile & n & \% \\
\hline Gender & & \\
\hline Female & 38 & 57.6 \\
\hline Male & 28 & 42.4 \\
\hline Age Group & & \\
\hline 20 to 30 & 55 & 83.3 \\
\hline 31 to 40 & 11 & 16.7 \\
\hline
\end{tabular}

Table 1: Demography of study population.

\begin{tabular}{|l|c|c|c|c|}
\hline \multirow{2}{*}{ Age Group } & \multicolumn{2}{|c|}{ Male } & \multicolumn{2}{c|}{ Female } \\
\cline { 2 - 5 } & $\mathbf{n}$ & $\mathbf{\%}$ & $\mathbf{n}$ & \% \\
\hline 20 to 30 & 23 & 82.1 & 32 & 84.21 \\
\hline 31 to 40 & 5 & 17.9 & 6 & 15.79 \\
\hline
\end{tabular}

Table 2: Age grouping statistics of study population.

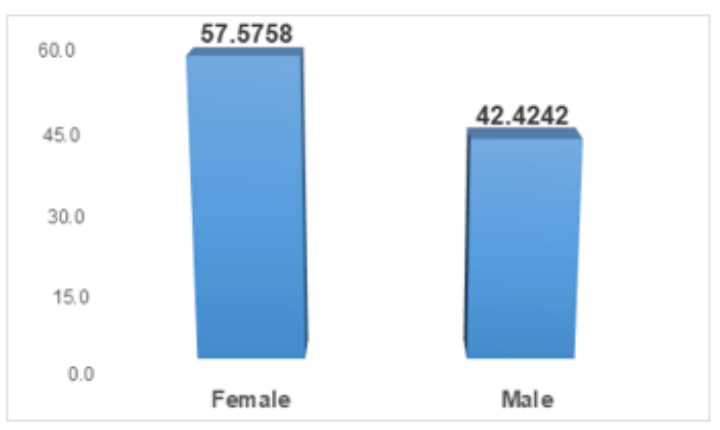

Figure 5: Showing gender profile of study population.

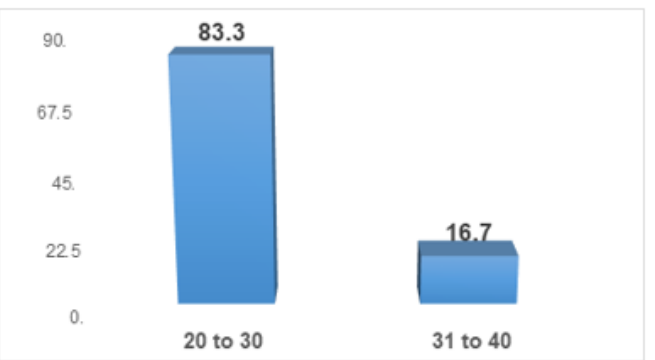

Figure 6: Showing age profile of study population. 
Furthermore, among female patients aged 31 to 40 years, results reveal that their mean laryngeal inlet is $1.164 \pm 0.184$, while a mean of $0.620 \pm 0.119$ for Glottis and $1.194 \pm 0.250$ for Tracheal air column.

Regardless of age, the resulting mean laryngeal, inlet, glottis and tracheal air column among female patients is $1.049 \mathrm{~cm}, 0.630$ $\mathrm{cm}$ and $1.131 \mathrm{~cm}$ respectively. All the measurements in female population are summarized in table 3 .

\begin{tabular}{|l|c|c|c|}
\hline Among Female & $\begin{array}{l}\text { Laryngeal } \\
\text { Inlet }\end{array}$ & Glottis & $\begin{array}{l}\text { Tracheal } \\
\text { Air Column }\end{array}$ \\
\hline $\begin{array}{l}\text { Among 20 to 30 } \\
\text { years old (n = 32) }\end{array}$ & & & \\
\hline Mean & 1.027 & 0.620 & 1.119 \\
\hline SD & 0.155 & 0.098 & 0.243 \\
\hline Median & 0.988 & 0.610 & 1.112 \\
\hline Range & 0.79 to 1.36 & 0.46 to 0.81 & 0.70 to 1.52 \\
\hline Among 31 to 40 & & & \\
\hline years old (n = 6) & & & \\
\hline Mean & 1.164 & 0.692 & 1.194 \\
\hline SD & 0.184 & 0.119 & 0.250 \\
\hline Median & 1.063 & 0.722 & 1.193 \\
\hline Range & 1.03 to 1.43 & 0.54 to 0.81 & 0.83 to 1.55 \\
\hline $\begin{array}{l}\text { Combined Age } \\
\text { Group (n = 38) }\end{array}$ & 1.049 & 0.630 & 1.131 \\
\hline Mean & 0.165 & 0.103 & 0.242 \\
\hline SD & 1.030 & 0.620 & 1.177 \\
\hline Median & & & \\
\hline Range & & & \\
\hline
\end{tabular}

Table 3: Summary of ultrasonography measurement of larynx (Female).

Results show that the average laryngeal inlet of male patients aged 20 to 30 years old is $1.19 \pm 2.26$ and a median of 1.19. Moreover, the mean glottis is $0.18 \mathrm{~cm} \pm 0.13$ while the resulting mean tracheal air column turns out to be $1.33 \pm 0.22$.

Furthermore, among male patients aged 31 to 40 years, results reveal that their mean laryngeal inlet is $1.33 \pm 0.18$, while a mean of $0.77 \pm 0.16$ for Glottis and $1.38 \pm 0.20$ for Tracheal air column.
Regardless of age, the resulting mean laryngeal, inlet, glottis and tracheal air column among male patients is $1.22 \mathrm{~cm}, 0.70 \mathrm{~cm}$, and $1.34 \mathrm{~cm}$ respectively. All the measurements in male population are summarized in table 4.

\begin{tabular}{|l|c|c|c|}
\hline Among Male & $\begin{array}{l}\text { Laryngeal } \\
\text { Inlet }\end{array}$ & Glottis & $\begin{array}{l}\text { Tracheal Air } \\
\text { Column }\end{array}$ \\
\hline $\begin{array}{l}\text { Among 20 to 30 } \\
\text { years old (n = } \\
\text { 23) }\end{array}$ & & & \\
\hline Mean & 1.19 & 0.18 & 1.33 \\
\hline SD & 2.26 & 0.13 & 0.22 \\
\hline Median & 1.19 & 0.69 & 1.33 \\
\hline Range & 0.89 to 1.62 & 0.47 to 0.92 & 0.93 to 1.72 \\
\hline $\begin{array}{l}\text { Among 31 to 40 } \\
\text { years old (n= 5) }\end{array}$ & 1.33 & & \\
\hline Mean & 0.18 & 0.16 & 0.20 \\
\hline SD & 1.37 & 0.79 & 1.34 \\
\hline Median & 1.06 to 1.56 & 0.54 to 0.99 & 1.14 to 1.69 \\
\hline Range & & & \\
\hline Combined Age \\
Group (n = 28)
\end{tabular}

Table 4: Summary of ultrasonography measurement of larynx (Male).

The graphical presentations of the measurement of Laryngeal Inlet, Glottis Opening and Tracheal Air Column (cm) both in male and female are shown in figure 7 and summary of all measurement data are shown in figure 8 (Male) and figure 9 (Female).

\section{Discussion}

Assessable upper airway extending from mouth to upper trachea is completely filled with air; it is difficult to assess their anatomy on sonography. However, it is possible to visualize various structures in relation to the upper airway whose anatomic location are either superficial or producing air-mucosa interface [8]. In general examination of the vocal cords and other internal laryngeal 


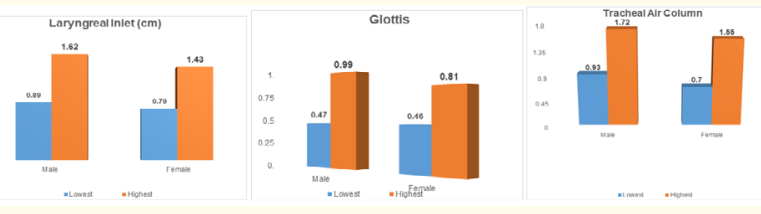

Figure 7: Graphical presentation of laryngeal inlet, glottis opening and tracheal air column $(\mathrm{cm})$.

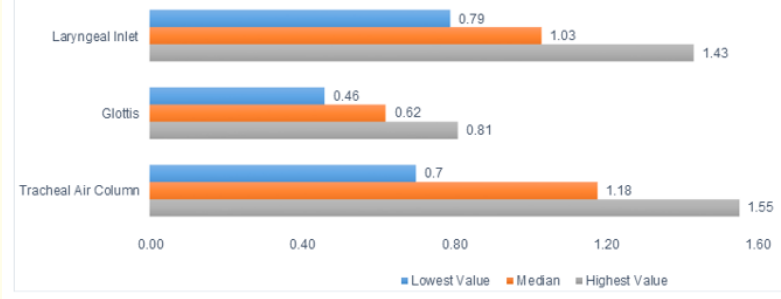

Figure 8: Summaries of data among all parameters (Among female).

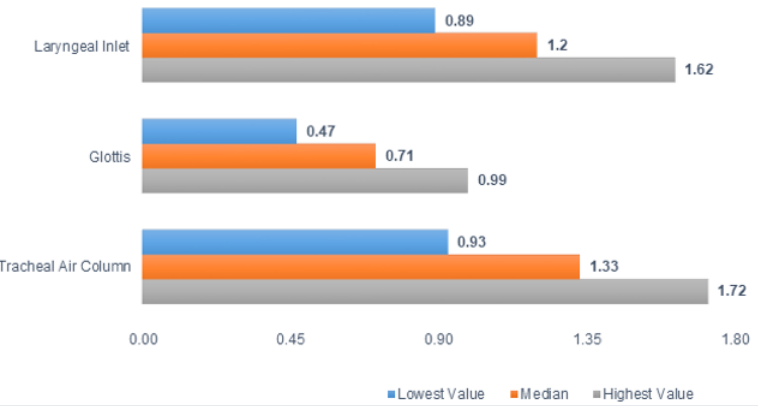

Figure 9: Summaries of data among all parameters (Among male).

structures are most commonly performed by indirect laryngoscope but this may not be tolerated by some patients' especially in children and old population or may not be possible immediately in certain locations where an otorhinolaryngologist opinion is not readily available. In those case we can perform quick assessment of larynx using sonography because of its easy availability and using high contrast interface between the mucosa lining of the upper air-way tract and the air-within it (an air-mucosa interface) as important landmark which has bright hyperechoic linear appearance [9].

Sonography is capable in providing detailed anatomic information and has numerous potential applications, as reported by Singh and Chan in their study of airway assessment in twentyfour (24) healthy volunteers [7]. It does not only provide a noninvasive way of anatomic investigation, but it can also show the dynamic aspect of the larynx. Ultrasound is safe, quick, noninvasive, repeatable and bedside tool to assess the airway and to describe its sonographic anatomy, as reported by Parmar and Mehta in their study of airway imaging in 100 healthy volunteers. In the study of Lakhal and Delplace involving nineteen (19) healthy volunteers, measurements of the subglottic diameter were performed adequately (and with precision of $0.33 \mathrm{~mm}$ ) and in a short period of time ( $<5$ minutes), making it a good alternative to MRI and CT scan and allows non-invasive assessment of the upper airway [10].

In addition, ultrasound proves to be helpful in pediatric patients with no need for sedation or general anesthesia to acquire images, as in CT or MRI examinations, which also are proven costly for our patients [11]. Hence, ultrasound appears as an easily reproducible and non-invasive way of investigating the structures of the larynx, which is feasible for all age group of patients [11].

The landmarks of larynx (hyoid bone, thyroid cartilage and cricoids cartilage) were easily visualized in this study and its sonographic appearance were all appreciated and demonstrated while scanning at transverse plane.

Hyoid bone was easily seen in transverse plane as hyperechoic inverted " $U$ " shaped structure with posterior acoustic shadow (Figure 1 and 6A). It has limited visibility at this level due to shadowing.

Thyroid cartilage appears as hypoechoic inverted ' $\mathrm{V}$ ' shaped structure (Figure 2 and 6B) [7]. It also provides the best window to view the vocal cords (Figure 2) [7]. The thyroid cartilage provided the best window for imagining the vocal cords. False and true vocal 
cords were visualized and easily distinguished from each other in all the participants by sliding the transducer in a cephalocaudad direction over the thyroid cartilage. The true vocal cord appeared as 2 triangular hypo echoic structures to its muscle content (vocalis muscle), outlined medially by the hyper echoic vocal ligaments (Figure 2) [7]. The false vocal cords lay parallel and cephalic to the true vocal cord and were more hyperechoic in appearance due to fatty nature (Figure 3) [7]. The true and false vocal cord could be further distinguished during phonation ("aa-aa" and "ee-ee" words) where the true vocal cords were observed to oscillate and move towards the midline compared with the false cords, which remained relatively immobile.

The cricoids cartilage is very interesting structure on Sonography which shaped like a signet ring structure and appeared hypoechoic. The circular configuration of the ring of cartilage was characteristic. The posterior lamina of the cricoids was obscured on sonography by air in the trachea, but the sides of the ring were easily visualized. These sides of the ring were seen as relatively hypoechoic structures with a homogeneous texture. The posterior surface of its anterior wall was delineated by a bright A-M interface as well as reverberation artifacts from intraluminal air (Figure 4 and 6C).

High frequency linear transducer has proven to be most appropriate in evaluating the larynx. This study has revealed the potential utility of ultrasound to assess the structural anatomy of larynx specially focusing at the hyoid bone level, thyroid cartilage level and cricoids cartilage level and creating normal values of laryngeal inlet, glottic opening and tracheal air column through sonography which can be used in various clinical applications. The measurement gained during this study was proven to be easily reproducible. Abduction and adduction of the vocal cords were very well visualized; hence we can add the value of ultrasound in the evaluation of larynx functionality. This can be great importance in some patient (i.e. singer, actors and teachers etc). Because of all these considerations, we conclude that ultrasound is a useful method in the evaluation of larynx.

\section{Conclusion}

Comparison with Computed tomography and magnetic resonance imaging would have helped in verifying that the measurements taken are accurate and ultrasound to be used as a modality in evaluation of the larynx. Limitation of laryngeal ultrasound is calcification of the thyroid cartilage with obscuration of distal structures especially in elderly individuals.

Laryngeal ultrasound is a reliable diagnostic tool on identifying different laryngeal anatomic structure, calculating various distances and areas with comparable accuracy to other previously used modalities as CT and MRI. In addition to the ability to diagnose laryngeal dynamic abnormality in patients with vocal fold palsy and partial laryngectomy. We recommend considering laryngeal ultrasound as initial method of screening in patient with query laryngeal injury either structural or functional (vocal cord palsy). Further work is also necessary to characterize the sonographic anatomy in patient with clinical abnormalities of the air-way. Here, a comparison with more established imaging techniques such as computed tomography and magnetic resonance imaging would be useful.

\section{Bibliography}

1. Friedman EM. "Role of ultrasound in the assessment of vocal cord function in infants and children". Annals of Otology, Rhinology and Laryngology: SAGE Journals 106.3 (1997): 199209.

2. Becker M., et al. "Imaging of the larynx and hypopharynx". European Journal of Radiology 66.3 (2008): 460-479.

3. Vats A., et al. "Laryngeal ultrasound to assess vocal fold paralysis in children". The Journal of Laryngology and Otology 118.6 (2004): 429-431.

4. Xia C., et al. "Usefulness of ultrasonography in assessment of laryngeal carcinoma". The British Journal of Radiology 86.1030 (2013): 20130343.

5. Dedecjus M., et al. "Real-time, high-resolution ultrasonography of the vocal folds-a prospective pilot study in patients before and after thyroidectomy". Langenbeck's Archives of Surgery 395.7 (2010): 859-864.

6. Ol'khova E., et al. "Laryngeal ultrasound studies: potential, prospects, limitations”. Vestn Otorinolaringol 5 (2009): 9-12.

7. Singh M., et al. "Use of sonography for airway assessment: an observational study". Journal of Ultrasound in Medicine 29.1 (2010): 79-85.

8. Kundra P., et al. "Ultrasound of the airway". Indian Journal of Anaesthesia 55.5 (2011): 456-462. 
9. Sites BD., et al. "Artifacts and pitfall errors associated with ultrasound-guided regional anesthesia. Part I: understanding the basic principles of ultrasound physics and machine operations". Regional Anesthesia and Pain Medicine 32.5 (2007): 412-418.

10. Lakhal K., et al. "The feasibility of ultrasound to assess subglottic diameter". Anesthesia and Analgesia 104.3 (2007): 611-614.

11. Garel C., et al. "Laryngeal ultrasonography in infants and children: anatomical correlation with fetal preparations". Pediatric Radiology 20.4 (1990): 241-244.

Volume 3 Issue 10 October 2021

(C) All rights are reserved by Sharma Nitu., et al. 